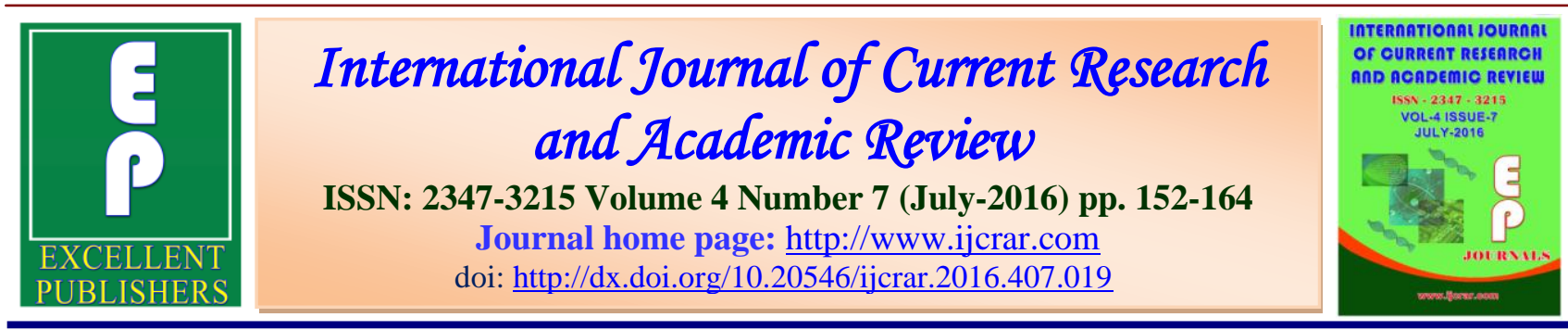

\title{
Antioxidant Activity of Lactococcus lactis and Curcumin Inhibits Lifespan Shorten and Oxidative Stress in Drosophila melanogaster
}

\section{SoJung Park ${ }^{1}$, WooSung Jeong ${ }^{2}$, KyungGon Kim ${ }^{3}$, Dae-Jung Kang ${ }^{4}$, Sang Yun Kim ${ }^{5}$ and Sang-Tae Kim $^{6} *$}

${ }^{1}$ Hankuk Academy of Foreign Studies, 50-Eydaero 54 Bungil Mohyenmaen Meongincity Keeonggeedo, 17035 Korea

${ }^{2}$ Whimoon High School, Yeoksamro 541, Kangnamgu 06183, Korea

${ }^{3}$ Asan Institute for Life Sciences, Asan Medical Center, Seoul, Department of Medicine, University of Ulsan College of Medicine, 43 gil Olympic-ro, Pungnapdong, Songpagu, Seoul 138-736, Republic of Korea

${ }^{4}$ Ildong Pharmaceutical Co. Ltd. 20 Samsung 1-ro, 1-gil, Hwaseong-si, Gyeonggi-do 18449, Korea

${ }^{5}$ Department of Neurology, Seoul National University College of Medicine, Goomi-ro, Bundanggu Seongnam city, Geonggeedo, 647-707 Republic of Korea

${ }^{6}$ W7, Biomedical Research Institute, Department of Neurology, Seoul National University College of Medicine, Healthcare Innovation Park3F, Goomi-ro, Bundanggu Seongnam city, Geonggeedo, 647-707 Republic of Korea

\section{*Corresponding author}

\section{KEYWORDS}

Oxidative stress;

Alzheimer disease;

life-span;

flight ability;

Lactococcus

lactis.
A B S T T R A C T

Oxidative stress has been strongly correlated with Alzheimer disease (AD) pathogenesis and the formation of intracellular $\mathrm{A} \beta 42 \mathrm{~h}$ as been reported in $\mathrm{AD}$ Drosophila model. In this study, the effect of curcumin and Lactococcus lactis was studied on lifespan, flight ability and oxidative stress in the AD Drosophila model flies and oxidative stress and protein carbonyl content were investigated in the fly brains. The progeny (AD flies) expressing human $\mathrm{A} \beta 42$ was exposed to 25,50 , and $100 \mu \mathrm{M}$ of curcumin or $2 \times 10^{5}, 2 \times 10^{6}$ and $2 \times 10^{7}$ of LAL in the diet for 7 days. The result showed that the treatment of flies to curcumin and LAL induced a dose dependent significant delay of lifespan, reduction in the oxidative stress and increase in the flight ability of AD Drosophila model. As a result, we concluded that curcumin and LAL can be potent in reducing $\mathrm{AD}$ symptoms. 


\section{Introduction}

Alzheimer's disease (AD) is an age-related neurodegenerative disease and the most common cause of dementia. The pathogenesis of $\mathrm{AD}$ is yet entirely clear and despite the increasing knowledge regarding the mechanism, no effective diseasemodifying therapy is yet available. Recently, many reports have shown to play a pivotal role in the synaptic damage, impairment of homeostasis, inflammation as well as toxicity in relation to $\mathrm{AD}$ etiology. Membranes can also be injury by the reactive oxygen species (ROS) that are produced by $\mathrm{A} \beta$ aggregates in the presence of metals such as copper, zinc or iron (Bush, 2003). Subsequent pathophysiological processes include mitochondrial damage (Abramov and Canevari, 2004), phosphorylated-Tau with consequent axonal transport damage and the trigger of cell death (Kienlen-Campard $\mathrm{P}$ and Miolet). However, until recently it has been impossible to take a global view to ask which biological processes are essential for the induction of the disease and which are downstream consequences of neurocytotoxicity (Cao and Song, 2008).

Knowing which biological processes are directly involved in initiating $\mathrm{AD}$ will allow us to key on those upstream targets that have the greatest therapeutic potential. Oxidative stress has been attributed as one of the important factors in progression of $\mathrm{AD}$ (Mubeenand Stephen, 2010). An importance has been given for the use of flavonoids to reduce the oxidative stress in the neurons (Christopher and Sylvain Doré,2011). Curcumin is the principal curcuminoid of the spice turmeric (Curcuma longa), a member of the ginger family (Figure 1) (Stagos and Amougias, 2012). Besides having a number of pharmacological properties (Siddique and Ara, 2012), in our earlier study it was reported to inhibit the induction of apoptosis in the AD model flies (Hong and Lee, 2012). In the present study, the effect of curcumin and Lactococcus lactis was studied on the life span, oxidative stress, and flight ability in the brains of transgenic Drosophila model of AD.

Lactococcus lactis (LAL) is an excellent source of dietary antioxidants. The scavenger potential of LAL in oxidative stress (OS) and vascular disease has been described and recent studies in Drosophila suggest that microbiota may be beneficial to individuals suffering from neuro degenerative diseases (Maity and Kumar, 2008). Therefore, in this study we describe the protective effects of curcumin and LAL concentrate on a transgenic Drosophila model of AD.

\section{Materials and Methods}

\section{Drosophia Strain}

Transgenic fly lines that expressed wild-type human $\mathrm{A} \beta 42$ under UAS control in neurons were obtained from Bloomington Drosophila Stock Center (Indiana University, Bloomington, IN). The progeny expressing the human $A \beta 42$ was generated by crossing males of UAS (Upstream Activation Sequence)-A $\beta 42$ strains with the females of GAL4-GMR (Ping and Hahm, 2015). Curcumin is purchased by SigmaAldrich (Gillingham, Dorset U.K). gQlab-S is friendly provided by Ildong pharmaceutical. Co. Ltd (Korea).

\section{Drosophila Culture}

The flies were cultured on standard Drosophila food containing $0.83 \%$ agar, $4.72 \%$ corn meal, $4.16 \%$ sugar, and $1.67 \%$ yeast at $25^{\circ} \mathrm{C}(24 \pm 1)$ (Siddique and Ara, 2012). Crosses were set up as described in earlier published work (Anterand Romero- 
Jiménez, 2011). The AD flies were exposed separately to different doses of curcumin (Sigma Aldrich, CAS 458-37-7) or LAL (Ildong pharmaceutical. Co. Ltd, Korea) and mixed in culture medium at final concentration of 25,50, and $100 \mu$ Mcurcumin and $2 \times 10^{5}, 2 \times 10^{6}$ and $2 \times 10^{7}$ LAL. The UAS-A $\beta 42$ acts as a control. The control flies were also separately exposed to the selected doses of curcumin and LAL. Longevity assays in the secondary screen and flies were reared on either $0.25 \%$ sucrose or LAL supplemented medium then collected under gaseous $\mathrm{CO}_{2}$ every 24 hours until a minimum of 50 adult females of each genotype were obtained. Briefly, flies were reared on either $0.25 \%$ sucrose or $5 \times 10^{5}$ or $5 \times 10^{8}$ cell number of LAL with $0.25 \%$ sucrose supplemented Tomato Juice medium then collected under gaseous $\mathrm{CO}_{2}$ every 24 hours until a minimum of 50 adult males of each genotype were obtained.

\section{Lifespan Determination}

For the determination of lifespan the newly enclosed female flies (control and AD) were placed in culture tubes (20 flies per tube) containing 25,50 , and $100 \mu \mathrm{M}$ of curcumin or $2 \times 10^{5}, 2 \times 10^{6}$ and $2 \times 10^{7}$ of LAL mixed in diet. The flies were transferred to new diet after every 4rd day and the number of dead flies was recorded at 3-day interval until the last one died (Abramoff and Magalhaes, 2004).

\section{Lipid Peroxidation Assay}

Lipid peroxidation test in the brain homogenate was determined according to the method described by Siddique et al., (Anoand Ozawa, 2015). Reagent1 (R1) was prepared by dissolving $64 \mathrm{mg}$ of 1-methyl-2phenylindole (MEP) into $30 \mathrm{ml}$ of acetonitrile to which $10 \mathrm{ml}$ of methanol was added to bring the volume to $40 \mathrm{ml}$. The preparation of $37 \% \mathrm{HCl}$ served as the reagent $\mathrm{R} 2$. The brains of flies were isolated under stereo zoom microscope in ice cold Tris- $\mathrm{HCl}(20 \mathrm{mM})$ (10 brains/group; five replicates/group). Homogenate was prepared in Tris- $\mathrm{HCl}$ and centrifuged at $4500 \mathrm{rpm}$ for $20 \mathrm{~min}$ and subsequently the supernatant was collected. In the tube $1300 \mu \mathrm{l}$ of R1 was taken. A volume of $1 \mu$ lwas added along with $300 \mu 1$ of $\mathrm{R} 2$ vortex and incubated at $45^{\circ} \mathrm{C}$ for $40 \mathrm{~min}$. After incubation, the tubes were cooled in on ice and centrifuged at $15,000 \mathrm{rpm}$ for $10 \mathrm{~min}$ at $4^{\circ} \mathrm{C}$ and read at $586 \mathrm{~nm}$.

\section{Estimation of Protein Carbonyl Content}

The protein carbonyl content was determined according to the method described by Hawkins et al. (Hawkins and Morgan, 2009). The brain homogenate was diluted to a protein concentration of approx. $1 \mathrm{mg} / \mathrm{ml}$. About $250 \mu \mathrm{l}$ of each diluted homogenate was taken in eppendorf centrifuge tubes separately. To it $250 \mu$ of $10 \mathrm{mM}$ 2,4-dinitrophenyl hydrazine (dissolved in $2.5 \mathrm{M} \mathrm{HCl}$ ) was added, vortexed, and kept in dark for $20 \mathrm{~min}$. About $125 \mu \mathrm{l}$ of $50 \%(\mathrm{w} / \mathrm{v})$ trichloroacetic acid (TCA) was added, mixed thoroughly, and incubated at $-20^{\circ} \mathrm{C}$ for $15 \mathrm{~min}$. The tubes were then centrifuged at $4^{\circ} \mathrm{C}$ for $10 \mathrm{~min}$ at $9,000 \mathrm{rpm}$. The supernatant was removed and the pellet obtained was washed twice on ice ethanol: ethyl acetate (1:1). Finally, the pellets were redissolved in $1 \mathrm{ml}$ of $6 \mathrm{M}$ guanidine hydrochloride and the absorbance was read at $370 \mathrm{~nm}$.

\section{Behavioral assays}

For the determine of climbing speed, groups of ten 3-day-old females were driven into 18-cm-long vials and incubated for $1 \mathrm{~h}$ at room temperature for environmental adaptation. After tapping the flies 
completely down to the bottom, we marked their climbing time at the $15-\mathrm{cm}$ finish line when more than five flies had arrived. Five trials were performed for each group and repeated with four different groups. The average climbing time was calculated for each genotype with standard error median value (SEM). Flight assay was performed as previously described (Pesah,2004) with 3day-old males $(\mathrm{n}>50)$.

\section{ROS formation measurement}

To measure Duox-dependent ROS formation in vivo, $\mathrm{H}_{2} \mathrm{O}_{2}$-specific Redox Sensor RedCC-1dye (Molecular probe) was used exactly as described previously (Tanaka and Matsumura,2002). The dissected guts of flies generating ROS sensor were fixed and images plated onto confocal dish for fluorescence analysis under ROI (relative of ratios) using LSM710 Confocal Microscope (Carl Zeiss, Germany)

\section{Statistical Analysis}

Statistical evaluation was used for lifespan expand, lipid peroxidation, estimation of protein carbonyl content, behavioral assay and ROS formation. Mean significant difference between treat groups was determined using one-way analysis of variance (AVOVA). The mean values of protein carbonyl contents, behavior analysis and lipid peroxidation assay of various fly groups were statistically compared using Student's $t$-test. The mean values of represent mean $\pm \mathrm{SE}$ of three experiments

\section{Results and Discussion}

Here, we studied a reduced lifespan in flies when of $A \beta 42$ expression is enhanced in the brain neurons using Drosophilamodel. The survival rate was measured only in female flies. As is evident from Fig. 2, the AD
Drosophila exposed to 25,50 , and $100 \mu \mathrm{M}$ of curcumin showed a dose dependent significant increase in the life span as compared to unexposed AD Drosophila. The control flies showed a life span of about 60 days. The median survival time of $A \beta 42-$ expressing Drosophila was reduced by $30 \%$ compared to wild type when both groups were fed a control diet. Interestingly, a diet rich in curcumin partially rescued the reduced lifespan caused by increased neuronal amounts of A $\beta 42$ in Drosophila (Fig. 2). A 342 -expressing Drosophila fed a diet containing $100 \mu \mathrm{M}$ of curcumin or $100 \mu \mathrm{M}$ of curcumin and $5 \times 10^{7}$ LAL had a 20-day $(30 \%)$ greater median lifespan than those fed a control diet. A concentration of $2 \times 10^{5}$ LAL dose was slightly observed the survival ratio withA $\beta 42$-expressing Drosophila. A dose dependent significant delay in the decreased of lifespan was observed in the AD Drosophila exposed to 25,50 , and $100 \mu \mathrm{M}$ of curcumin or $2 \times 10^{5}$, $2 \times 10^{6}$ and $2 \times 10^{7}$ of LAL(Fig. 2). The results obtained for the determination of lifespan are shown in Fig. 1. The AD Drosophila exposed to 25,50 , and $100 \mu \mathrm{M}$ of curcumin or $2 \times 10^{5}, 2 \times 10^{6}$ and $2 \times 10^{7}$ of LALshowed a dose dependent significant delay in the shorten of lifespan as compared to unexposed AD Drosophila and control Drosophila.

Viability the number of pupae of filial generation of Drosophila exposed to the low dose $(25 \mu \mathrm{M})$ of curcumin was not different $(125 \%)$ from those in the control group $(P<$ 0.05). The number of pupae of filial generation of Drosophila exposed to the high dose $100 \mu \mathrm{M}$ of curcumin was dramatically lower $(51 \%)$ than those in control and low dose curcumin group. Meanwhile, the number of pupae of filial generation of Drosophila exposed to the low dose $\left(2 \times 10^{5}\right.$ of LAL) was not different $(113 \%)$ from those in the control group 
$(P<0.05)$. The number of pupae of filial generation of Drosophila exposed to the high dose $2 \times 10^{7}$ of LAL was dramatically lower $(83 \%)$ than those in control and low dose curcumin group. Also, the number of pupae of filial generation of Drosophila exposed to the high dose $\left(2 \times 10^{7}\right.$ of LAL and $100 \mu \mathrm{M}$ of curcumin) was dramatically lower $(46 \%)$ than those in control and low dose curcumin group. The data collected for the female flies byfly lifespan was observed by survival ratio (group $(P<0.005)$. For control flies the number of survival were more compared to AD flies. Lethality of pupae in group of low concentration $25 \mu \mathrm{M}$ of curcumin (8\%) was slightly lower than in control group (17\%). The number of pupae of filial generation of Drosophila exposed to the high dose $100 \mu \mathrm{M}$ of curcumin was slightly lower $(16 \%)$ than those in control and low dose curcumin group $(P<0.05)$. In LAL, lethality of pupae in group of low concentration $\left(2 \times 10^{5}\right)(10 \%)$ was slightly lower than in control group (11\%). The number of pupae of filial generation of Drosophila exposed to the high dose $2 \times 10^{7}$ of LAL was slightly higher $(7 \%)$ than those in control and low dose curcumin group $(P<0.005)$ (Fig. 3).

No change in the lethality of control flies exposed to 25,50 , and $100 \mu \mathrm{M}$ of curcumin was observed (Fig. 3). Lethality of pupae in group of low concentration of $25 \mu$ Mcurcumin was slightly lower than in control group (Fig. 3). The results obtained for the assay of lipid peroxidation are shown in Fig. 4. The AD flies treated to 25, 50, and $100 \mu \mathrm{M}$ of curcumin showed a dose dependent significant decrease in the lipid peroxidation as compared to unexposed AD Drosophila and control Drosophila (Fig. 4).

Also, the AD Drosophila treated to $2 \times 10^{5}$, $2 \times 10^{6}$ and $2 \times 10^{7}$ of LAL showed a dose dependent slight decrease in the lipid peroxidation as compared to unexposed AD Drosophila and control Drosophila (Fig. 4). The results obtained for the assay of flight ability are shown in Fig.5. The AD Drosophila exposed to 25,50 , and $100 \mu \mathrm{M}$ of curcumin or $2 \times 10^{5}, 2 \times 10^{6}$ and $2 \times 10^{7}$ of LAL showed a dose dependent significant increase in the flight ability as compared to unexposed AD Drosophila and control Drosophila (Fig. 5).

Final analysis in this study, the results obtained for protein carbonyl content are shown in Fig. 6. A dose dependent significant decrease in the mean absorbance values was obtained in $\mathrm{AD}$ flies exposed to 25,50 , and $100 \mu \mathrm{M}$ of curcumin or $2 \times 10^{5}$, $2 \times 10^{6}$ and $2 \times 10^{7}$ of LAL as compared to unexposed AD Drosophila and control Drosophila (Fig. 6). The unexposed AD Drosophila showed the highest mean absorbance value as compared to control Drosophila(Fig. 6).

We further analyzed that ROS is abolished in the UAS-A $\beta 42 / G M R-G a l 4$ flies, indicating that DUOX is required for $A \beta 42-$ induced ROS generation and bacteria population. To observe the DUOX activation in vivo, we generated transgenic flies that generated ROS by $A \beta 42$ expression, because activated DUOX is known to be localized in the membrane region of the intestine cells. Using these flies, we observed the A $\beta 42$ expression activates ROS formation in gut (Fig. 7 and 8, upper panel right), but the low level of ROS formation observed in curcumin and LAL-treated flies was completely restored to the w118 flies level (Fig. 7 and 8, middle and bottom panel). Importantly, curcumin and LAL were abolished in the UAS-A $342 /$ GMR-Gal4 flies, indicating that DUOX is boosted for A $\beta 42$-induced ROS formation. Furthermore, A $\beta 42$-induced ROS formation by curcumin and LAL treatment was abolished in UAS- 
A $342 / G M R-G a l 4$ flies. These results demonstrated that $A \beta 42$ are boosted for DUOX-expressing intestines but curcumin and LAL is sufficient to retard all of the ROS formation necessary for DUOXdependent gut immunity that is required for resisting pathogen infection.

\section{Conclusions}

The results of the present study revealed that the exposure of AD Drosophila to 25, 50, and $100 \mu \mathrm{M}$ of curcumin showed a dose dependent significant increase in the lifespan, reduction in lipid peroxidation, protein carbonyl content, and increase in the flight ability. Oxidative stress as a result of the accumulation of $A \beta 42$ has been reported in neurons of AD Drosophila model (Favrin and Bean, 2013). It remains still unclear that the degenerating neuron itself or misfolded proteins directly causes toxicity during the progression of AD (Janet and Helmfors, 2012). In our primary studies with the same Drosophila models, various plant extracts and flavonoids have been reported to delay the loss of flight ability and reduced oxidative stress (Jeong and Kim,2014).

Fig.1 Chemical structure of curcumin.<smiles>COc1cc(/C=C/C(=O)CC(=O)/C=C/c2ccc(O)c(OC)c2)ccc1O</smiles>

Fig.2 Effect of curcumin and LAL on survival rate measured in transgenic Drosophilain various treated groups. The concentration indicated with $100 \mu$ Mcurcumin, 2x107 of LAL.

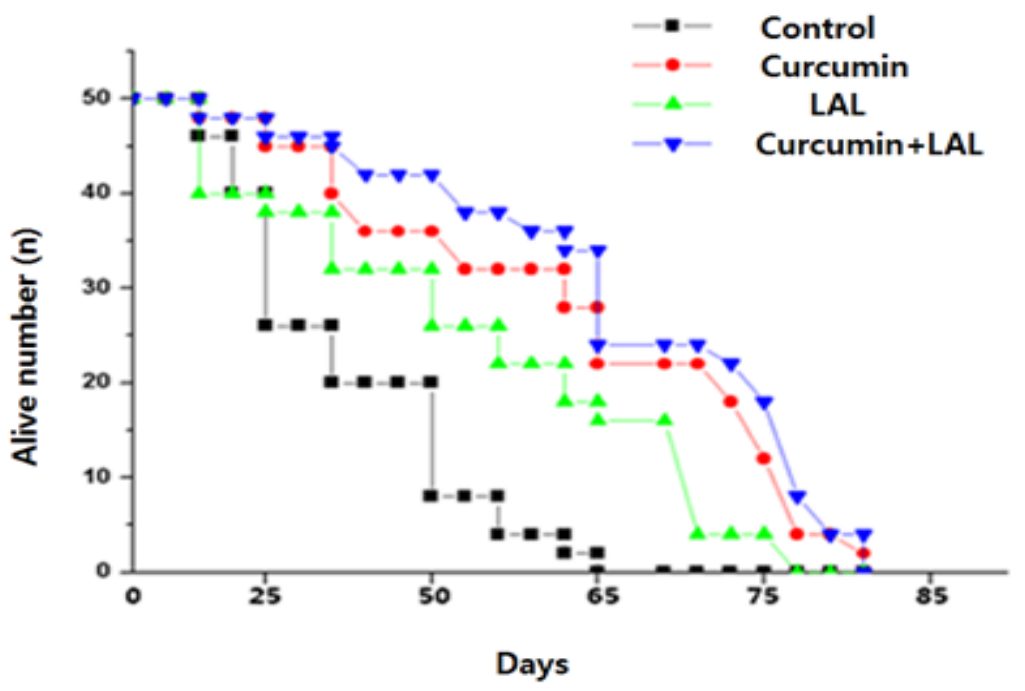


Int.J.Curr.Res.Aca.Rev.2016; 4(7): 152-164

Fig.3 Curcumin and LAL effect on quantity of pupae in filial generation. Quantity of pupae per vial (three pair of parents). Ten vials per experimental group. Mean $\pm \mathrm{SEM} ;{ }^{*} \mathrm{P}<0.05$ : curcumin $100 \mu \mathrm{M}$ vs control and curcumin $25 \mu \mathrm{M}$ and curcumin $50 \mu \mathrm{M}$. $* * \mathrm{P}<0.01$ : $2 \times 107$ of LALvs control and 2x105 and 2x106 of LAL.

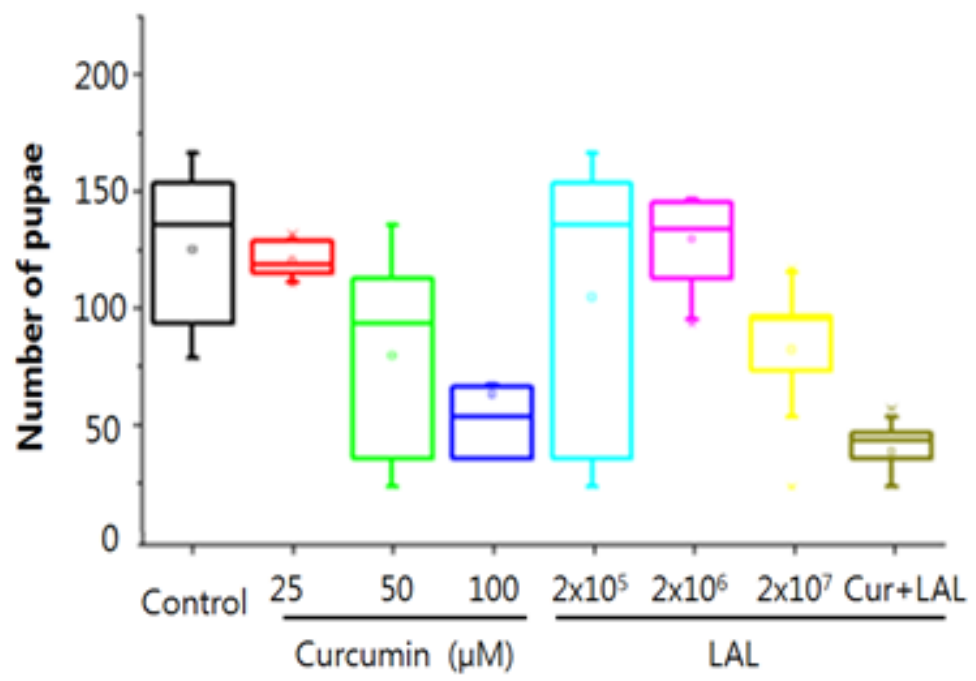

Fig.4 Curcumin (a) and LAL (b) effect on the lethality of pupae. ${ }^{*} \mathrm{P}<0.05$ : curcumin $25 \mu \mathrm{M}$ vs control, $* * \mathrm{P}<0.01$ : curcumin $50 \mu \mathrm{M}$ vs control and curcumin $100 \mu \mathrm{M}$. $* \mathrm{P}<0.05$ : LAL $2 \times 105$ vs control, $* * \mathrm{P}<0.01$ : LAL 2x106 vs control and 2x107 LAL

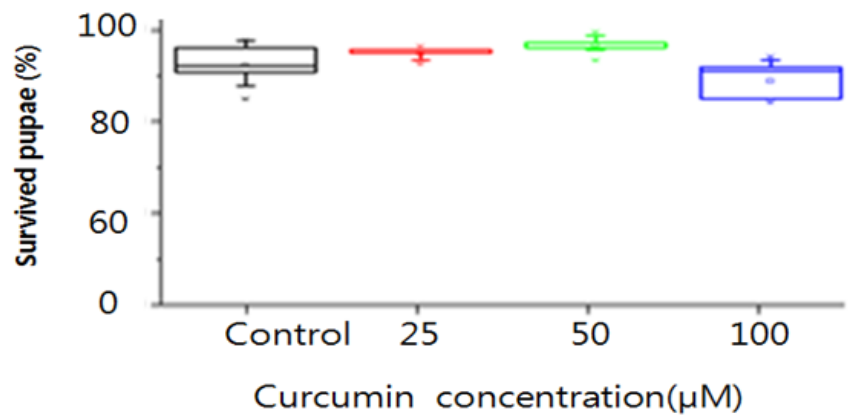

a)

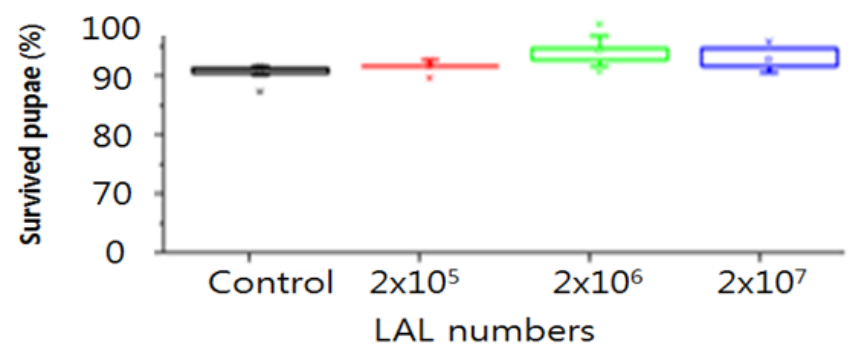

b) 
Fig.5 Curcumin (a) and LAL (b) effect on lipid peroxidation measured in the brains of transgenic Drosophila after 24 days of the exposure in treated groups. $(\mathrm{C} 1=25 \mu \mathrm{M}$ curcumin; $\mathrm{C} 2=50 \mu \mathrm{M}$ curcumin; $\mathrm{C} 3=$ curcumin $100 \mu \mathrm{M})$; * Significant with respect to control, $\mathrm{P}<0.05$; **Significant with respect to AD model flies, $\mathrm{P}<0.01$; (C4=2x105 LAL; C5=2x106 LAL; C6=2x107 LAL). \# Significant with respect to AD model flies, $\mathrm{P}<0.005)$.

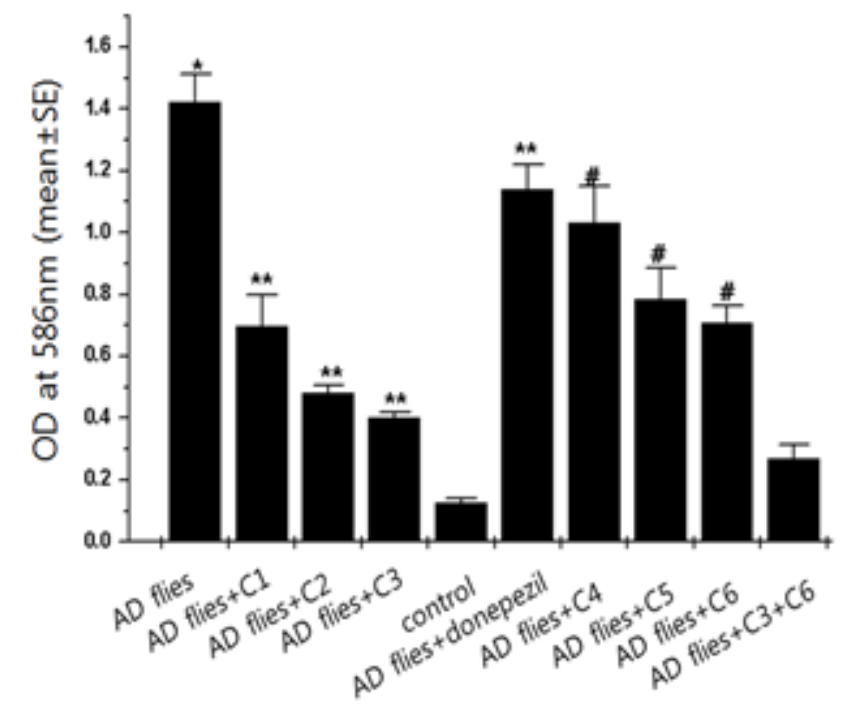

Fig.6 Curcumin and LAL effect on vertical flight ability. Flight index \% of flies moved to the top vial; mean \pm SEM; $\mathrm{P}<0.05$ : Curcumin vs control group, two ways ANOVA, Bonferroni adjusted.

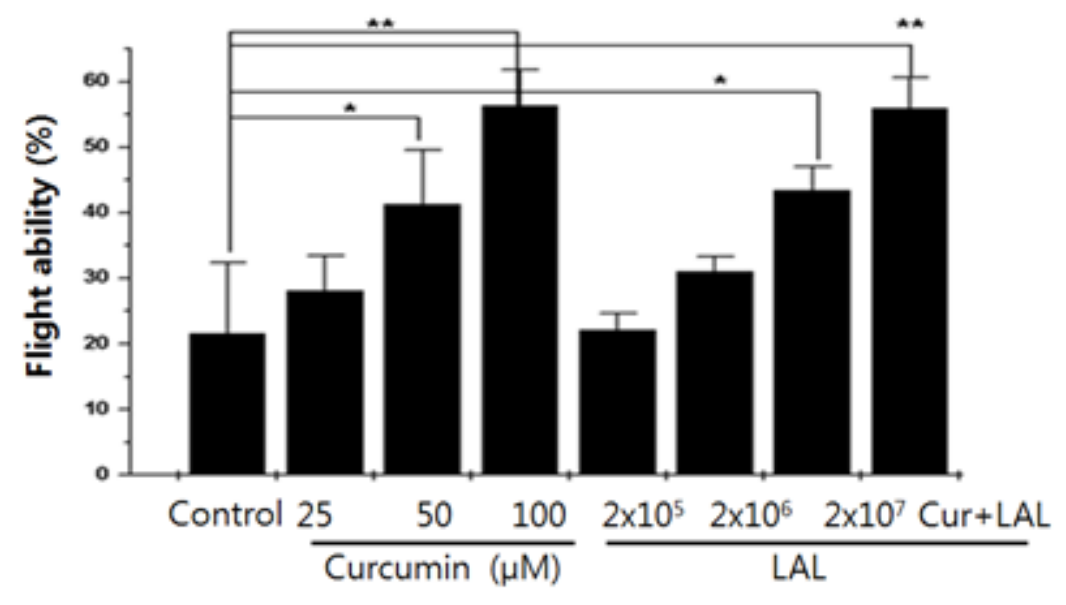


Fig.7 Effect of curcumin and LAL on protein carbonyl content measured in the brains of transgenic Drosophila after 24 days of the exposure in various treated groups. $(25 \mu \mathrm{M}, 50 \mu \mathrm{M}$ and $100 \mu \mathrm{M}$ curcumin; a significant with respect to $\mathrm{AD}$ model, $* \mathrm{P}<0.05$; a significant with respect to $\mathrm{AD}$ model flies, $* \mathrm{P}<0.05) .(25 \mu \mathrm{M}, 50 \mu \mathrm{M}$ and $100 \mu \mathrm{M}$ curcumin; a significant with respect to control, $* * \mathrm{P}<0.01$; significant with respect to AD Drosophila model, $* * \mathrm{P}<0.01$ ). (LAL $2 \times 105$; LAL 2x106; LAL 2x107; a significant with respect to AD Drosophila model, $\mathrm{P}<0.05$; significant with respect to $\mathrm{AD}$ Drosophila model, $\mathrm{P}<0.01$ ).\# $\mathrm{P}<0.005$, significant with respect to $\mathrm{AD}$ Drosophila model, a significant with respect to AD Drosophila model, $\# \mathrm{P}<0.005$, donepezil vs AD Drosophila model. Standard control used with $100 \mu \mathrm{g} / \mathrm{ml}$ donepezil.

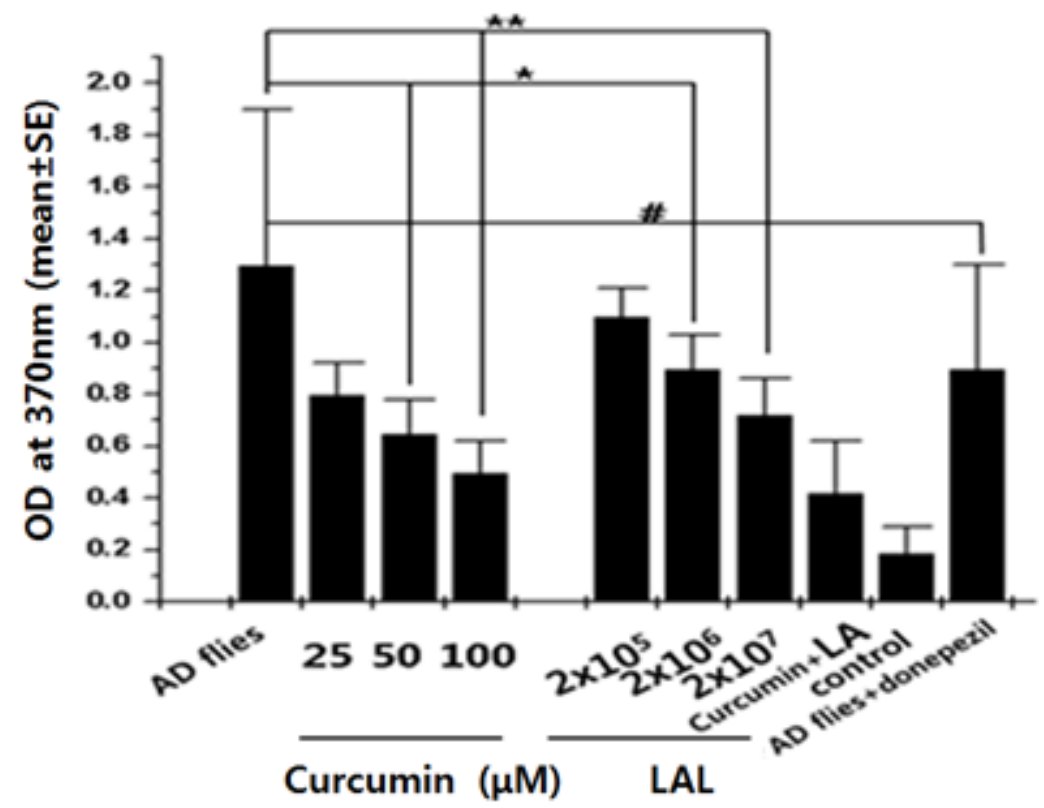

Fig.8 Curcumin and LAL treatment was repressed with ROS formation in UAS-A $\beta /$ GMR-Gal4 flies. All flies (3days-old) were orally treated with $100 \mu \mathrm{M}$ curcumin or LAL $2 \times 107$ for $24 \mathrm{~h}$ and Duox dependent ROS formation in the midgut was visualized by $\mathrm{H} 2 \mathrm{O} 2$-specific RedoxSensor RedCC-1dye (red). Representative Confocal microscopic images (a) and percentage of ROSpositive intestines were shown (b). Data were analyzed using an AVOVA analysis and values represent mean mean \pm SEM $(* \mathrm{P}<0.05, * * \mathrm{P}<0.01, \# \mathrm{P}<0.005)$ of at least three independent experiments.

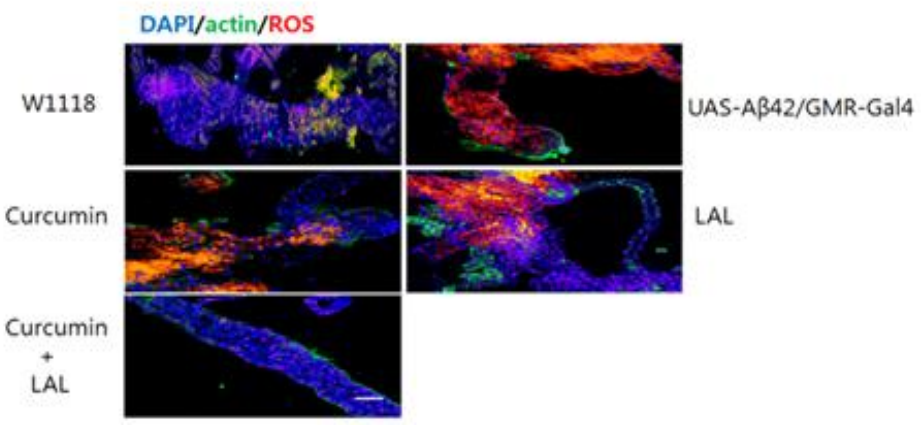




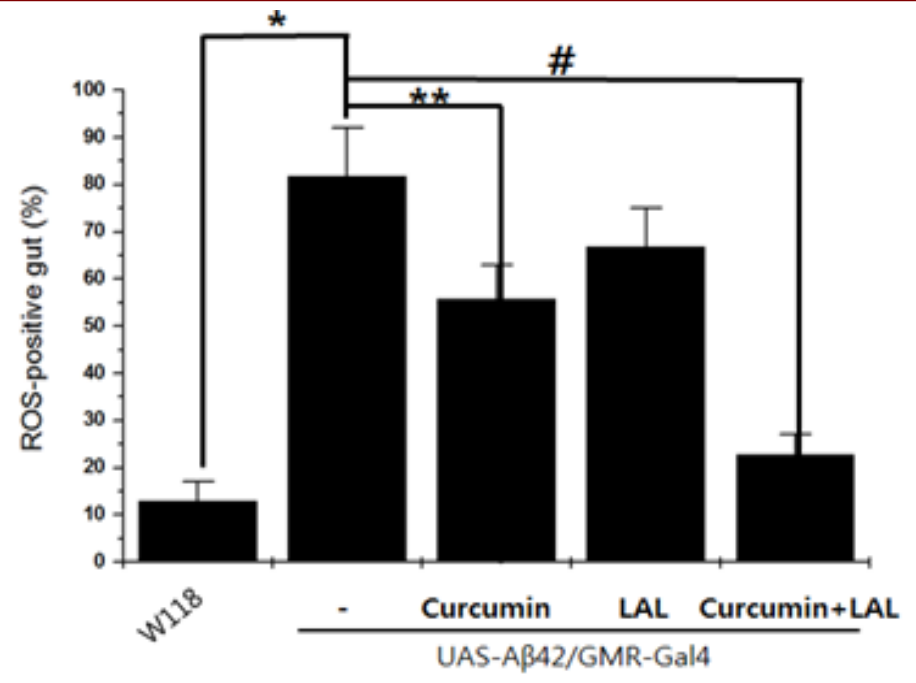

b)

Flavonoids have been reported to show improvements in cognition function possibly by protecting vulnerable neurons or by stimulating neuronal regeneration (Zhang and Ruolph, 2012). In present study, treatment of curcumin has shown reduction in lipid peroxidation and protein carbonyl content in the brains of AD Drosophila model. This protection is attributed to an antioxidant nature of curcumin (Caesar and Jonson, 2012). Recent findings have suggested that flavonoids have a remodeling effect on the nature of curcumin, converting them into nontoxic, smaller amorphous aggregates, thus preventing the formation of reactive oxygen species (Rona, 2014). On the other hand, an antioxidant nature of the curcumin is attributed to its unique conjugated structure that includes two methoxylated phenols (Barzegar, 2011). It has been reported to inhibit the generation of ROS responsible for DNA and membrane damage (Grabowska and Kucharewicz, 2015). Although the animals are well acquainted with the self-defense mechanism, an enhancement in stress beyond the capacity of an animal to cope up may result in cellular damage leading to the cell death (Ray and Bisht, 2011). In previous reports, curcumin has shown the neuro-protection in the A $\beta 42$ Drosophila model due to its antioxidant potential and its capability to penetrate into the brain (Jeremy and Liu, 2014). It has been reported to alleviate A $\beta 42$-induced toxicity, reduce ROS level, and protect cell against apoptosis (James and Barbara, 2010). The aggregation of $A \beta 42$ in the brain has been implicated as a crucial step in the formation of plaques and curcumin has anti-fibrillogenic and fibrildestabilizing properties, thus inhibiting the formation of $\mathrm{A} \beta 42$ fibrillar or plaques (Siddique and Smita, 2014). In D. melanogaster, curcumin have been reported to extend life span in a gender and genotype specific manner (Shen and Peng, 2013). In present study, the life span and flight ability were studied on female AD Drosophila. There are reports on the life span extension of curcumin in mice and Caenorhabditi selegans (Luisa and Stefania, 2013). This extension is due to the neuro-protective, lifespan and lipid peroxidation properties of curcumin (Seongand Lee, 2015). The therapies involving natural antioxidants/ plant products may be used as adjunct therapy. These results obtained in our present study and our primary study, in 
which the watermelon and LAL was studied using the same AD fly strain, results in neuro-protective effects (Koand Eun, 2014). The present study was carried out usingA $\beta 42$-induced AD Drosophila model and consequent flight dysfunction. The this Drosophila model mimics the neuronal injury associated with AD and can be used to study whether or not a variety of natural compounds or medicines mixed in the fly feds have the neuroprotective potential.

Thus far, several previous studies have highlighted curcumin and LAL as a main player in regulating AD. For instance, AD inhibits lifespan shorten by regulating curcumin or LAL in a mice model and an AD model (35-39). With this in vivo Drosophila model organism, the data presented here add additional evidence supporting curcumin and LAL emerging role as a potentially attractive agent that could prevent AD. Taken together, in this report, the novel effects of curcumin and LAL on regulating lifespan and its capacity to mitigate environmental oxidative stresses and flight ability are described using Drosophila as an in vivo model organism. Considering that it has become one of most widely administered well-being food supplements in Korea and in western countries, this report would spur further research to discover curcumin and LAL poorly characterized health benefits, thereby eventually helping pave new avenues to utilize curcumin and LAL as a key component in medical regimens in order to prevent and cure many forms of neuronal degeneration.

\section{References}

Abramov A.Y. ,Canevari L.,Duchen M.R.Beta-amyloid peptides induce mitochondrial dysfunction and oxidative stress in astrocytes and death of neurons through activation of NADPH oxidase.
J. Neurosci. 2004.14.24(2): 565-575.

Abramoff M.D., Magalhaes P.J., Ram C.J. Image Processing with Image. Biophotonics International. 2004. 11(7): 36-42.

Ano Y., Ozawa M.,KutsukakeT., Sugiyama S., Uchida K., Yoshida A., Nakayama H. Preventive effects of a fermented dairy product against Alzheimer's disease and identification of a novel oleamide with enhanced microglial phagocytosis and anti-inflammatory activity. PLoS One2015. 10(3): e0118512.

Anter J., Romero-Jiménez M.,FernándezBedmar Z.,Villatoro-Pulido M.,Analla M., Alonso-Moraga A., Muñoz-Serrano A.Antigenotoxicity, cytotoxicity, and apoptosis induction by apigenin, bisabolol, and protocatechuic acid. $\mathbf{J}$ Med Food. 2011. 14(3): 276-83.

Bush A.I. The metallobiology of Alzheimer's disease.Trends Neurosci. 2003. V. 26(4): 207-214.

BarzegarA..Ali A., Moosavi-Movahedi. Intracellular ROS Protection Efficiency and Free Radical- Scavenging Activity of Curcumin.PLoS One. 2011. 6(10): e26012.

Caesar I., Jonson M., Peter K., Nilsson S., Thor PerHammarström. CurcuminPromotes A-beta Fibrillation and Reduces Neurotoxicity in Transgenic Drosophila. PLoS One. 2012. 7(2): e31424.

Cao W., Song H. J., Gangi T.,Kelkar A.,Antani I., Garza D., Konsolaki M. Identification of novel genesthat modify phenotypes induced by Alzheimer's $\beta$ amyloid overexpression in Drosophila. Genetics. 2008. 178(8): 1457-1471.

Christopher C., Sylvain Doré D.L. Dietary flavonoids are neuroprotective through Nrf2-coordinated induction of endogenous cytoprotective proteins. NutrNeurosci. 2011. 14: 226-236.

Favrin G., Bean D.M.,Bilsland E., Boyer H., Fischer B.E., Russell E.,Crowther D.C.,Baylis, B.A. Oliver,

S.G.; Giannakou, M.E. Identification of novel 
modifiers of $A \beta$ toxicity by transcriptomic analysisin the Fruitfly.Sci Rep.2013. 16(3): 3512.

Filipčík P.,Cente M.,Gerenčík M.,Hulín I., and Novák M.The role of oxidative stress in the pathogenesis of Alzheimer's disease," Bratisl. Med J.2006.vol. 107(9-10).384-394.

Grabowska W.,Kucharewicz K.,Wnuk M., Lewinska A., Suszek M.,Przybylska D., Mosieniak G., Sikora G., BielakZmijewska A.Curcumin induces senescence of primary human cells building the vasculature in a DNA damage and ATM-independent manner. Age. 2015. 37(1): 9744.

Hawkins C.L., Morgan P.L., Davies M.J. Quantification of protein modification by oxidants. Free Radical Biology and Medicine. 2009.46(8):965-988.

James A., Barbara Shukitt-Hale J., Gregory J., Karen A., Kalt W.W., Fisher D.R.Differential protection among fractionated blueberry polyphenolic families against DA-, Abeta(42)- and LPS-induced decrementsin $\mathrm{Ca}(2+)$ buffering in primary hippocampal cells. J Agric Food Chem. 2010. 58(14): 8196-8204.

Janet R.,Helmfors L., Williams J., Leila M.,Menzer L.,Dumoulin M., David A., Lomas C., Damian M., Ann-Christin Brorsson D. Disease-related amyloidogenic variants of human lysozyme trigger the unfolded protein response and disturb eye development in Drosophila melanogaster. FASEB J. 2012. 26(1): 192-202.

Jeong J.H., Kim H.J., Park S.K., Jin D.E., Kwon O.L., Kim H.J.,Heo H.L.An investigation into the ameliorating effect of black soybean extract on learning and memory impairment with assessment of neuroprotective effects. BMC Complement Altern Med. 2014. 14(14):56.

Jeremy E., Liu C.K., Yan X.,Toldo S., Selden T.,María M.E., Matthew I.R., Ye H.D.,Zang S. Discovery of 5-(4-
Hydroxyphenyl)-3-oxo-pentanoic Acid (2-(5-Methoxy-1H-indol-3-yl)-ethyl)amide as a Neuroprotectant for Alzheimer's Disease by Hybridization of Curcumin and Melatonin.ACS Chem Neurosci. 2014. 20.5(8): 690-699.

Kienlen-Campard P.,Miolet S.,Tasiaux B., Octave J.N. Intracellular amyloid-beta 1-42, but not extracellular soluble amyloid-beta peptides, induces neuronal apoptosis. J. Biol. Chem. 2002. 277(18): 15666-15670.

Ko D.G., Eun, Y.B., Na J.U., KiMS.T. "Dual PINK Mutant and Aß42-Dependent Lifespan Shorten and Flight Impairment in Transgenic Drosophila Partially Alleviates by a Lactococcus lactis Supplemented

Diet,"Journal of Behavioral and Brain Science.2015.vol. 92:266-273.

Luisa D., Stefania R., Margherita R., Massimo S., Mario S. Oleuropein Aglycone Protects Transgenic C.elegans Strains Expressing A $\beta 42$ by Reducing Plaque Load and Motor Deficit.PLoS One. 2013. 8(3):e58893 .

Maity T. K., Kumar R.,Misra A.K. Prevalence of Enteropathogenic Escherichia coli Isolated from Chhana Based Indian Sweets in Relation to Public Health. Indian J Microbiol. 2009. 50(4): 463-7.

Mubeen A., Stephen W. Oxidative Stress in the Progression of Alzheimer Disease in the Frontal Cortex. J Neuropathol ExpNeurol, 2010. 69(2):155-167.

Pesah Y.Drosophila parkin mutants have decreased mass and cell size and increased sensitivity to oxygen radical stress. Development. 2004. 131(9): 2183-2194.

Ping Y.,Hahm E.T.,Waro G., Song Q., Vo-Ba D.A.,Licursi A.,Bao H.,Ganoe L., Finch, K., Tsunoda S. Linking A $\beta 42$-induced hyperexcitability to neurodegeneration, learning and motor deficits, and a shorter lifespan in an Alzheimer's model. PLoS Genet. 2015.16. 11(3):e1005025.

Ray B.,Bisht S.,Maitra A.,Maitra 
A.,LahiriD.L.Neuroprotectiveand

Neurorescue Effects of a Novel Polymeric Nanoparticle Formulation of Curcumin (NanoCurc ${ }^{\mathrm{TM}}$ ) in the Neuronal Cell Culture and Animal Model: Implications for Alzheimer's Disease. J Alzheimers Dis. 2011. 23(1): 61-77.

Rona Banerjee. Inhibitory Effect of Curcumin$\mathrm{Cu}$ (II) and Curcumin-Zn(II) Complexes on Amyloid-Beta Peptide Fibrillation. Bioinorg Chem Appl. 2014. 325873.

Seong K. M., Lee Y., Park M., Jin S.H., MinY. W. Curcumin Mitigates Accelerated Aging after Irradiation in Drosophila by Reducing Oxidative Stress.Biomed Res Int.2015.425380.

Siddique Y.H.,Ara G.,Jyoti S.,Afzal M. Protective effect of curcumin in transgenic Drosophila melanogaster model of Parkinson's disease. J Diet Suppl. 2012. 9(1): 1-8.

Stagos D.,Amougias G.,Matakos A.,Spyrou A.,Tsatsakis A.M., Kouretas D. Chemoprevention of liver cancer by plant polyphenols, Food and Chemical Toxicology. 2012.50(6):2155-2170.

Susana G.,Guzmán-Beltrán G., MedinaCampos O. N.,Pedraza-Chaverri J. Curcumin Pretreatment Induces Nrf2 and an Antioxidant Response and Prevents Hemin-Induced Toxicity in Primary Cultures of
Cerebellar Granule Neurons of Rats," Oxid Med Cell Longev. 2013. 801418.

Tanaka H., Matsumura I.,Ezoe S., Satoh Y.,Sakamaki T., Albanese C.,Machii T.,Pestell R.,Kanakura Y. E2F1 and cMyc potentiate apoptosis through inhibition of NF- $\mathrm{KB}$ activity that facilitates MnSOD-mediated ROS elimination. Mol. Cell. 2002. 9(5): 1017-1029.

Wang P., Su C., Li R., Wang H.,Ren Y., Sun H., Yang J., Sun J., Shi J.,Tian J., Jiang S. Mechanisms and effects of curcumin on spatial learning and memory improvement in APPswe/PS1dE9 mice. J Neurosci Res. 2014. 92(2): 218-31.

Wei W., Norton D.D., Wang X.,Kusiak J. W. Abeta 17-42 in Alzheimer's disease activates JNK and caspase- 8 leading to neuronal apoptosis. Brain. 2002. 125(9): 2036-2043.

Yin H., Zhou Y., Wen H., Zhou C., Zhang W., Hu X., Wang L., You C.,Shao J. Curcumin sensitizes glioblastoma to temozolomide by simultaneously generating ROS and disrupting AKT/mTOR signaling. Oncol Rep. 2014. 32(4): 1610-6.

Zhang C.,Ruolph E.Natural Modulators of Amyloid-Beta Precursor Protein Processing. Curr Alzheimer Res. 2012.26: 13.

\section{How to cite this article:}

SoJung Park, WooSung Jeong, KyungGon Kim, Dae-Jung Kang, Sang Yun Kim and Sang-Tae Kim. 2016. Antioxidant Activity of Lactococcus lactis and Curcumin Inhibits Lifespan Shorten and Oxidative Stress in Drosophila melanogaster. Int.J.Curr.Res.Aca.Rev.4 (7): 152-164. doi: http://dx.doi.org/10.20546/ijcrar.2016.407.019 